\title{
The special case of the three body problem, when gravitational potential is given as the Kislik potential.
}

\author{
A. Shuvalova ${ }^{1}$ and T. Salnikova ${ }^{2}$ \\ ${ }^{1}$ Lomonosov Moscow State University, Moscow, 119991, Russia \\ email: a.shuvalova@yahoo.com \\ ${ }^{2}$ Lomonosov Moscow State University, Moscow, 119991, Russia \\ email: tatiana.salnikova@gmail.com
}

\begin{abstract}
In this paper we consider the special case of the planar circular restricted three-body problem by the example of the problem of the Earth, the Moon and a point mass, where the gravitational potentials of the Earth and the Moon are given as the Kislik potential. The Kislik potential takes into account the flattening of a celestial body on the poles. We find the relative equilibria solutions for a point mass and analyze their stability. We describe the difference between the obtained points and the classical solution of the three-body problem.
\end{abstract}

Keywords. celestial mechanics, methods: analytical, three-body problem, libration point, flattening, gravitational potential.

\section{Introduction}

In classical celestial mechanics for most cases it is quite enough to research the problem when the actual attracting masses are considered as material points. This situation can be easily explained by the fact that the size of attracting bodies compared with the distances between them is usually small enough to neglect. Completely different situation is observed in celestial ballistics. Sometimes the spacecraft is located near the body, and the distance between them is comparable with the size of the body. In this case it is necessary to consider the shape of the body. Therefore the ways of representing the gravitational potential of the planet are of interest from a practical point of view. A variety of main problems of celestial ballistics has been described in the middle of the 20th century. Those problems are connected with the motion of a spacecraft in the noncentral gravitational field. The differential equations of motion are the following:

$$
\ddot{x}-2 \omega \dot{y}-\omega^{2} x=U_{x}^{\prime}, \quad \ddot{y}+2 \omega \dot{x}-\omega^{2} x=U_{y}^{\prime}, \quad \ddot{z}=U_{z}^{\prime}, \quad U=\frac{\gamma m}{r}+\mu R(x, y, z, t, \mu),
$$

where $\gamma$ is the gravitational constant, $r$ is the position vector of a moving point, $\omega$ is the angular velocity of the rotating coordinate system, $\mu R$ is the perturbation function, $\mu$ is a small parameter. Potential of the planet $U$ can be written as

$$
U=\frac{\gamma m}{r}+\sum_{k=2}^{\infty} J_{k}(R / r)^{k} P_{k}(z / r),
$$

where $P_{k}$ is the Legendre polynomial, $R$ is the average equatorial radius of the planet, $J_{k}$ is the constant. $J_{2}$ characterizes the flattening of the planet, $J_{3}$ characterizes the asymmetry of the planet relative to the equatorial plane $\left(J_{2} \sim-10^{-3}, J_{3} \sim 10^{-5}\right)$. 
It is known that L. Euler has reduced to the quadratures the problem of the motion of the point mass in the gravitational field of two fixed points. This idea gets a new important application for the theory of the artificial satellites of the Earth. M. D. Kislik was the first who proposed to use the problem of two fixed masses to describe the gravitational potential. In 1961 V. G. Dyomin has showed that the potential of the two fixed points can represent the gravitational field of flattened planet with high accuracy (Dyomin (1968)). The potential can be determined by the following formula

$$
U=\frac{\gamma m}{2}\left(\frac{1+i \delta}{r_{1}}+\frac{1-i \delta}{r_{2}}\right), \quad r_{1,2}=\sqrt{x^{2}+y^{2}+(z-c(\delta \pm i))^{2}} .
$$

Constants $c$ and $\delta$ characterize the shape of the planet. Thus, if a celestial body is compressed along its axis of dynamic symmetry, we can assume that the gravitational potential is approximated by the potential of two points with complex conjugate masses located at the imaginary distance, the gravitational potential is still a real value. If we know the constants $J_{2}$ and $J_{3}$ from (1.2), then $c$ and $\delta$ can be expressed as

$$
c=\frac{\sqrt{-J_{3}^{2}-4 J_{2}^{3}}}{2 J_{2}} R, \quad \delta=\frac{J_{3}}{\sqrt{-J_{3}^{2}-4 J_{2}^{3}}} .
$$

When $\delta=0$, the formula (1.3) does not take into account the asymmetry of the planet relative to the equatorial plane. In this case the form of gravitational potential corresponds to the Kislik potential.

\section{Overview}

In this paper we consider the special case of the planar circular restricted three-body problem by the example of the problem of the Earth (E), the Moon (M) and a point mass $(\mathrm{P})$, where the gravitational potentials of $\mathrm{E}$ and $\mathrm{M}$ are given as the Kislik potential.

Let us consider the planar circular restricted three-body problem. We neglect the mass of the P. E and M have masses $m$ and $m-\mu$, respectively, and perform circular motion around their barycenter $O$. The rotating coordinate system is as usual: $O$ is the origin of coordinates, x-axis goes through E and M. E, M and P move in the plane $z=0 . \vec{\omega}=\omega \overrightarrow{e_{z}}$ is the angular velocity of the moving coordinate system. If we take the Kislik potential in the Earth-Moon problem, $\omega$ satisfies the following equation:

$$
\omega^{2}=\frac{\gamma(m-\mu)}{\left(\left(x_{E}+x_{M}\right)^{2}-c^{2}\right)^{3 / 2}}+\frac{\gamma \mu}{\left(\left(x_{E}+x_{M}\right)^{2}-\lambda^{2} c^{2}\right)^{3 / 2}} .
$$

To simplify the analytical calculus let us take the classical solution of the two-body problem:

$$
\omega^{2}=\gamma m /\left(x_{E}+x_{M}\right)^{3} .
$$

Then the gravitational potentials of $\mathrm{E}$ and $\mathrm{M}$ take the form

$$
U_{E}=\frac{\gamma(m-\mu)}{\sqrt{\left(x+x_{E}\right)^{2}+y^{2}-c^{2}}}, \quad U_{M}=\frac{\gamma \mu}{\sqrt{\left(x-x_{M}\right)^{2}+y^{2}-\lambda^{2} c^{2}}},
$$

where $\lambda$ is the constant coefficient. Motion of $\mathrm{P}$ is determined by the system of equations (1.1). Constant $c$ for $\mathrm{E}$ is equal to $209.9 \mathrm{~km}$. Ellipticity of $\mathrm{M}$ is 3 times less than ellipticity of $\mathrm{E}$, the radius of $\mathrm{M}$ is equal to 0.273 radius of $\mathrm{E}$. So in the numerical calculations we assume that $\lambda=0$. We define the effective potential energy as

$$
V_{\omega}=-\frac{1}{2} \omega^{2}\left(x^{2}+y^{2}\right)-U_{E}-U_{M}
$$



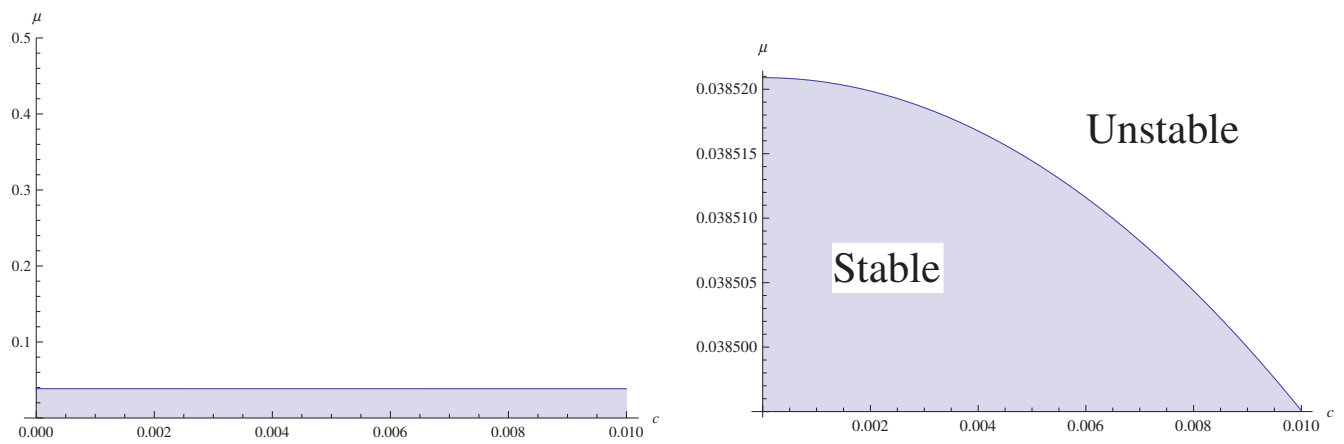

Figure 1. On the figure there is shown dependence of $\mu$ on $c$, if $\lambda=0$. If $c=0$, the condition for the stabilization $L_{4}$ and $L_{5}$ is the same as the condition for the stabilization in the classical three-body problem, i.e. $\mu<0,0385209$.

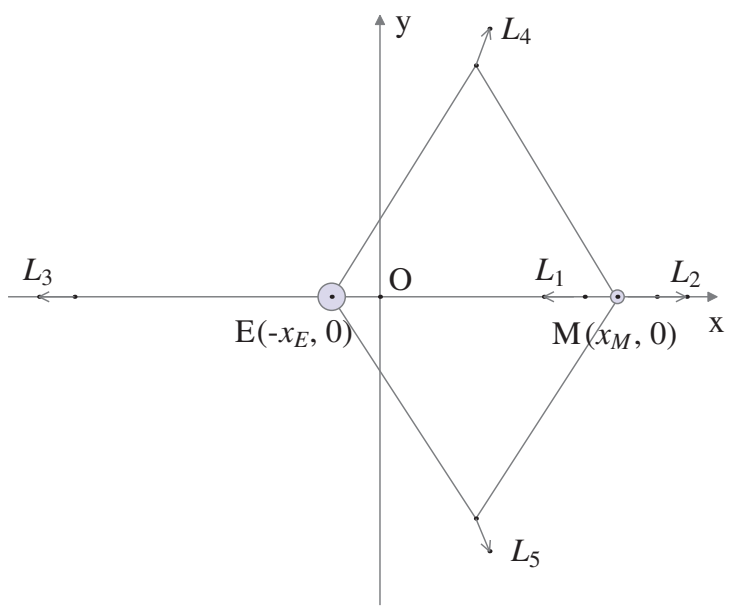

Figure 2.

Libration point. Relative equilibrium equations are

$$
\begin{aligned}
\omega^{2} x & =\frac{\gamma\left(x+x_{E}\right)(m-\mu)}{\left(\left(x+x_{E}\right)^{2}+y^{2}-c^{2}\right)^{3 / 2}}+\frac{\gamma\left(x-x_{M}\right) \mu}{\left(\left(x-x_{M}\right)^{2}+y^{2}-\lambda^{2} c^{2}\right)^{3 / 2}}, \\
\omega^{2} y & =\frac{\gamma y(m-\mu)}{\left(\left(x+x_{E}\right)^{2}+y^{2}-c^{2}\right)^{3 / 2}}+\frac{\gamma y \mu}{\left(\left(x-x_{M}\right)^{2}+y^{2}-\lambda^{2} c^{2}\right)^{3 / 2}} .
\end{aligned}
$$

If $y=0$, we have 3 unstable libration points $L_{1,2,3}$ as in the usual three-body problem. $L_{1}, L_{2}$ and $L_{3}$ can be obtained from the equation:

$$
\omega^{2} x=\frac{\gamma\left(x+x_{E}\right)(m-\mu)}{\left(\left(x+x_{E}\right)^{2}-c^{2}\right)^{3 / 2}}+\frac{\gamma\left(x-x_{M}\right) \mu}{\left(\left(x-x_{M}\right)^{2}-\lambda^{2} c^{2}\right)^{3 / 2}} .
$$

The second member function has 4 vertical asymptotes $\left(x=-x_{E} \pm c, x=x_{M} \pm \lambda c\right)$ and decreases monotonically.

If $y \neq 0$, then the coordinates of $\mathrm{P}$ can be found analytically, the coordinates of points $L_{4}$ and $L_{5}$ satisfy the equations: $\left(x+x_{E}\right)^{2}+y^{2}=\left(x_{E}+x_{M}\right)^{2}+c^{2},\left(x-x_{M}\right)^{2}+y^{2}=$ $\left(x_{E}+x_{M}\right)^{2}+\lambda^{2} c^{2}$. In the classical solution of the three-body problem $E M L_{4}$ and $E M L_{5}$ satisfy the equations: $\left(x+x_{E}\right)^{2}+y^{2}=\left(x_{E}+x_{M}\right)^{2},\left(x-x_{M}\right)^{2}+y^{2}=\left(x_{E}+x_{M}\right)^{2}$. 
If we assume that $x_{E}+x_{M}=1, m=1, \mu=\widetilde{\mu}$, the condition for the stabilization of $L_{4}$ and $L_{5}$ in the first approximation takes the following form:

$$
1-27 \widetilde{\mu}+27 \widetilde{\mu}^{2}+9 c^{4}\left(1+\left(-1+\lambda^{4}\right) \widetilde{\mu}\right)+6 c^{2}\left(-1-2\left(1+2 \lambda^{2}\right) \widetilde{\mu}+3\left(1+\lambda^{2}\right) \widetilde{\mu}^{2}\right)>0 .
$$

On Fig. 1 there is shown the dependence of $\widetilde{\mu}$ on $c$, if $\lambda=0$.

Our calculations give us the value of the shift of each libration point. It approximately equals to $0.05 \mathrm{~km}$. Fig. 2 schematically shows the directions of the shifts of the libration points from their positions in the classical problem.

\section{Conclusion}

Thus, we can conclude that in the problem considered above, the shape of the bodies does not provide substantial changes in the solutions. But the Earth is not the most flattened planet of the Solar system.

The value of $c$ was calculated for several planets:

\begin{tabular}{|c|c|}
\hline & $\mathrm{c}, \mathrm{km}$ \\
\hline Earth & 209.9 \\
Mars & 150.013 \\
Jupiter & 8461.57 \\
Saturn & 7547.368 \\
\hline
\end{tabular}

The polar flattening of Jupiter is equal to 0.065 (0.0033 for the Earth), the flattening of Saturn is equal to 0.1 . These values are greater, so in the problem, where we use a gravitational potential according to our algorithm for these planets, we can expect more visible effect.

\section{References}

Demin, V. G., 1968, The motion of an artificial satellite in the noncentral gravitational field, Nauka, Moscow.

Markeev, A. P., 1978, The libration points in celestial mechanics and space dynamics, Nauka, Moscow.

Worthington, J., 2012, A Study of the Planar Circular Restricted Three Body Problem and the Vanishing Twist, Thesis, Univ. Sydney. 